\title{
Das Verstärkermodell der Suizidalität: Chronische Suizidalität bei der Borderline- Persönlichkeitsstörung verstehen und behandeln
}

\author{
Johannes M. Hennings \\ Einheit für dialektisch-behaviorale Therapie, kbo-Isar-Amper-Klinikum München-Ost, Haar/München, Deutschland
}

\section{Schlüsselwörter}

Borderline-Persönlichkeitsstörung · Dialektisch-behaviorale Therapie · Nichtsuizidale Selbstverletzung · Suizidalität · Verstärkung

\begin{abstract}
Zusammenfassung
Trotz großer Fortschritte in der evidenzbasierten Psychotherapie wird unser Versorgungssystem durch chronisch suizidale Patienten mit einer Borderline-Persönlichkeitsstörung (BPS) weiterhin stark herausgefordert. Die BPS ist mit einem hohen Suizidrisiko von 5-10\% sowie einem hohen Selbstverletzungsrisiko von bis zu $80 \%$ behaftet. Therapeuten wie Angehörige fühlen sich oft überfordert und hilflos, wenn sie mit der Suizidalität der Patienten konfrontiert sind. Immer wieder kommt es so zu Therapieabbrüchen, Vorstellungen in Notaufnahmen oder akut-psychiatrischen Einweisungen. Bei der nichtsuizidalen Selbstverletzung (NSSV) - einem Verhaltensmuster, das gehäuft zusammen mit chronischer Suizidalität bei BPS auftritt - tragen Verstärkermechanismen (z.B. Nachlassen von Anspannung) dazu bei, dass sich Borderline-Patienten trotz längerfristig unangenehmen Folgen immer wieder selbst verletzen. Die Motive für NSSV und suizidales Verhalten können sehr unterschiedlich sein. Es spricht jedoch einiges dafür, dass die zugrunde liegenden Mechanismen ähnlich sind und sich hieraus wichtige therapeutische Interventionsmöglichkeiten ableiten lassen. In dieser Übersichtsarbeit werden die Hintergründe und Unterschiede von NSSV, Suizidideationen sowie Suizidversuchen bei chronisch suizidalen Borderline-Patienten dargestellt. Neuere Erkenntnisse der modernen Neuro- und Verhaltenswissenschaft werden in ein Verstärkermodell der Suizidalität
\end{abstract}

integriert, von dem verhaltenstherapeutische Interventionen abgeleitet werden, die Therapeuten in ihrer Arbeit mit chronisch suizidalen Patienten unterstützen können.

(c) 2021 The Author(s)

Published by S. Karger AG, Basel

\section{The Reinforcement Model of Suicidality: Understanding and Psychotherapy of Chronic Suicidality in Borderline Personality Disorder}

\author{
Keywords \\ Borderline personality disorder - Dialectic-behavioral \\ therapy · Non-suicidal self-injury · Suicidality · \\ Reinforcement
}

\section{Abstract}

In spite of great advancements in evidence-based therapies, chronic suicidal patients with borderline personality disorder (BPD) still challenge our mental health system. BPD is afflicted with a high suicide risk of $5-10 \%$ and a high risk for self-mutilation (up to $80 \%$ ). Therapists as well as relatives feel often stunned and helpless when confronted with suicidality resulting in interruption of therapies, repeated presentations to emergency rooms and referrals to hospitals. Reinforcement mechanisms turned out to play an important role in the maintenance of repeated non-suicidal self-injury (NSSI). While individual motives for NSSI and suicidal behavior including suicidal ideations can differ, the principal mechanisms appear to be transferrable. This article aims to give a better idea of karger@karger.com www.karger.com/ver

Karger ${ }^{\prime \prime}=$

BOPEN ACCESS (c) 2021 The Author(s)

Published by S. Karger AG, Basel

This is an Open Access article licensed under the Creative Common Attribution-NonCommercial-4.0 International License (CC BY-NC) (http://www.karger.com/Services/OpenAccessLicense), applicable to the online version of the article only. Usage and distribution for commercial purposes requires written permission.
Korrespondenz an:

Johannes M. Hennings, johannes.hennings@ kbo.de 
what is behind and what are the differences between non-suicidal self-injury, suicidal ideations and suicide attempts. It further integrates recent developments of behavioral science in a reinforcement model of suicidality that can provide therapists a practical armamentarium in their work with chronic suicidal clients.

(c) 2021 The Author(s)

Published by S. Karger AG, Basel

\section{Einleitung}

Suizidalität ist eine der schwerwiegendsten Symptome psychischer Störungen. Sie umfasst psychopathologische Phänomene, die von Lebensüberdruss, Suizidideationen (Gedanken an und Vorstellungen vom eigenen Tod, aber auch Abwägen und Planen eines Suizides), ambivalenten Suizidversuchen, Suizidversuchen (SV) bis hin zum vollendeten Suizid reichen [Linehan, 1986; Klonsky et al., 2016]. Patienten mit einer emotional instabilen Persönlichkeitsstörung (BPS) haben ein besonders hohes Risiko für Suizide (5-10\%), SV, sowie Selbstverletzungen (bis zu $80 \%$ ), die mitunter klinisch schwer von einem SV zu differenzieren sind [Klonsky, 2007; Klonsky et al., 2013]. Zudem liegt bei BPS oft eine sogenannte chronische Suizidalität vor. Auch wenn dieser Begriff zur Einschätzung der Suizidalität wegen seiner Unschärfe psychopathologisch nicht gebraucht werden soll [Wolfersdorf, 2008], beschreibt er sehr treffend, wie Klienten oft über viele Jahre immer wieder oder anhaltend unterschiedliche Formen der Suizidalität erleben [Oumaya et al., 2008]. Diagnoseübergreifend sind mittlerweile robuste Risikofaktoren für suizidales Verhalten herausgearbeitet worden, insbesondere Missbrauch in der Kindheit, nichtsuizidale Selbstverletzung (NSSV) sowie frühere SV [Klonsky et al., 2013; Franklin et al., 2017]. Allerdings entstammen diese Risikofaktoren vorwiegend aus Fall-Kontroll-Studien und helfen dem Kliniker wenig, das individuelle Suizidrisiko zu einem bestimmten Zeitpunkt zuverlässig zu bestimmen [Linehan et al., 2012; Miller et al., 2017; Teismann et al., 2019]. Auch, wenn der Großteil der Betroffenen ihren Suizidideationen nicht nachgehen werden [Joiner, 2005], können wir "Suizidideatoren" und spätere "Suizidversucher" anhand selbst der besten Indikatoren (z.B. Angst vor dem Tod, subjektive Schmerztoleranz, objektiv anhaltender Schmerz) nicht zuverlässig voneinander trennen [Paashaus et al., 2019]. Und tatsächlich sind die Suizidraten in bestimmten Populationen, wie z.B. bei jugendlichen Mädchen, in den letzten Jahren wieder angestiegen [Curtin et al., 2016; Miller et al., 2017]. Auf der anderen Seite ist es für chronisch suizidale Borderline-Klienten oft sehr schwer, einen Therapieplatz zu bekommen oder zu behalten. Stattdessen werden sie immer wieder wegen ihrer Suizidalität in Kliniken eingewiesen, wobei wenig Evidenz vorliegt, dass die Unterbrechung von Therapien, Kran- kenhauseinweisungen oder geschlossene Unterbringungen Suizidalität überhaupt senken [Linehan et al., 2012]. Es fehlt uns außerdem an evidenzbasierten pharmakologischen Behandlungsmöglichkeiten für Borderline-Patienten [Lieb et al., 2004]. Hierzu passt wiederum, dass $\mathrm{Pa}$ tienten mit einer BPS oft wiederholte psychiatrische Krankenhausaufenthalte und Therapieabbrüche aufweisen, und weiterhin zu denjenigen mit der höchsten Rate an Suizidideationen, NSSV und wiederholten SV gehören [Linehan et al., 1983; Linehan, 1993].

Diese Übersicht beschäftigt sich daher mit der Frage, was die Hintergründe chronischer Suizidalität sind und welche psychologischen Mechanismen sie aufrechterhalten. Sind die Modelle, die für das Verstehen von NSSV entwickelt wurden, auf suizidales Verhalten übertragbar? Können oder sollten wir überhaupt mit suizidalen Patienten Psychotherapie machen? Und wenn ja, welche Interventionen sind hilfreich?

\section{Hintergründe von NSSV bei der BPS}

NSSV (z.B. Schneiden, Ritzen, Kopf Anschlagen, Verbrennen) ist ein weltweites Phänomen, das nicht nur, aber besonders häufig bei BPS vorkommt [Plener et al., 2009]. Sie ist definiert als eine selbstintendierte, sozial unangemessene Verletzung der Körperoberfläche ohne suizidale Absicht [Linehan et al., 2006]. Um das Phänomen in Studien besser untersuchen zu können, hat sich diese einheitliche Definition mittlerweile durchgesetzt und ist in der 5. Ausgabe des Diagnostic and Statistical Manual of Mental Disorders sogar als eine neue diagnostische Entität gewürdigt worden [American Psychiatric Association, 2013]. In der kürzlich erschienen Meta-Analyse von Taylor et al. [2018] zu NSSV wurde eine Reihe unterschiedlicher psychologischer Funktionen von NSSV zusammengestellt. Zu den häufigeren, sogenannten intrapsychischen Funktionen zählen Emotionsregulation und Selbstbestrafung, wobei es darum geht, (aversive) innere Zustände zu beenden oder zu verhindern. Weniger häufig sind sogenannte interpersonelle Funktionen wie z.B. Einflussnahme auf andere oder Zugehörigkeit zu Peers [Klonsky, 2007; Klonsky et al., 2013; Taylor et al., 2018]. In ähnlicher Weise unterscheiden Nock und Prinstein [2004] in ihrem 4-Funktionen-Modell im Sinne der kognitiven Verhaltenstherapie positive (i.e., Zuführen eines angenehmen Stimulus) versus negative (i.e., Wegnahme eines aversiven Stimulus) sowie automatische (i.e., intrapersonelle; z.B. Emotionsregulation wie oben) versus soziale (i.e., interpersonelle; e.g., Aufmerksamkeit, Vermeidung, Flucht) Verstärker (Tab. 1). Durch diesen Erklärungsansatz ist es leichter nachzuvollziehen, warum einige Patienten sich immer wieder selbst verletzen und dabei in Kauf nehmen, sich in chirurgischen Notaufnahmen vorstellen zu müssen (was oft sehr schambesetzt ist), dass Konflikte mit Angehörigen entstehen (was erneute 
Tabelle 1. Vier-Funktionen-Modell der NSSV (modifiziert nach Nock und Prinstein [2004])

\begin{tabular}{|c|c|}
\hline Positive Verstärkung & Negative Verstärkung \\
\hline $\begin{array}{l}\text { Automatisch-positive Verstärkerfunktion von NSSV: } \\
\rightarrow \quad \text { Um einen gewünschten physiologischen Zustand } \\
\text { herzustellen (i.e., Emotionen hervorrufen, Emotionen nicht } \\
\text { abzuschwächen), z.B.: } \\
\text { - Selbstbestrafung } \\
\text { - Sensationslust } \\
\text { - Identitätsgefühl ("das gehört zu mir") } \\
\text { - Sexuelle Befriedigung } \\
\text { - Kontrolle/Handlungsfähigkeit }\end{array}$ & $\begin{array}{l}\text { Automatisch-negative Verstärkerfunktion von NSSV: } \\
\rightarrow \quad \text { Um Anspannung oder andere aversive Affektzustände } \\
\text { abzuschwächen, z.B.: } \\
\text { - Abschwächen von aversiven Gefühlen } \\
\text { - Anspannungsreduktion } \\
\text { - Antidissoziativ } \\
\text { - Symptome anderer psychischer Störungen reduzieren } \\
\quad \text { (z.B. Zwangsgedanken, Stimmenhören) }\end{array}$ \\
\hline $\begin{array}{l}\text { Sozial-positive Verstärkerfunktion von NSSV: } \\
\rightarrow \quad \text { Als eine Form der Kommunikation mit und/oder ohne } \\
\text { Einflussnahme auf andere, z.B.: } \\
\text { - Kommunikation von Leiden } \\
\text { - Erhalten von Zuwendung und Aufmerksamkeit } \\
\text { - Veranlassen bestimmter Maßnahmen (z.B. Medikation } \\
\quad \text { erhalten; nicht nach Hause entlassen werden) }\end{array}$ & $\begin{array}{l}\text { Sozial-negative Verstärkerfunktion von NSSV: } \\
\rightarrow \quad \text { Um Verantwortungen abzugeben, Konsequenzen oder } \\
\text { zwischenmenschliche Herausforderungen zu } \\
\text { vermeiden, z.B.: } \\
\text { - Sich selbst stellvertretend für andere verletzen } \\
\text { - Sich selbst verletzen, bevor man durch andere verletzt } \\
\quad \text { wird }\end{array}$ \\
\hline
\end{tabular}

Krisen provoziert) und dass stigmatisierende Narben zurückbleiben.

$\mathrm{Zu}$ diesem psychologischen Verstärkermodell passen gut neuere neurobiologische Beobachtungen, die im Bereich des limbischen Systems gemacht wurden: In einer fMRT-Studie reduziert NSSV die Aktivität der Amygdala bei Borderline-Patienten und verbessert gleichzeitig die Konnektivität zum Gyrus frontalis superior [Reitz et al., 2015]. Klinisch zeigt sich durch die NSSV eine sofortige Abnahme aversiver Anspannung und die Betroffenen können wieder klarer denken. Darüber hinaus aktiviert NSSV das Belohnungssystem, wobei Strukturen des Opioid- und wahrscheinlich auch Endocannabinoid-Systems beteiligt sind [Kirtley et al., 2015]. In einer prospektiven Studie mit Borderline-Patienten, die sich mit einer hohen Frequenz selbst verletzten, erfassten Houben et al. [2017] über Palmtop-Geräte kontinuierlich die emotionalen Zustände über mehrere Tage. Hier zeigte sich ein enger Zusammenhang zwischen dem Auftreten von aversiven Gefühlszuständen mit einer darauffolgenden Selbstverletzung. Erstaunlicherweise konnte so nicht nur die NSSV mit einer hohen Wahrscheinlichkeit vorhergesagt werden, sondern auch ein darauffolgender, aversiver Gefühlszustand nach einer NSSV (z.B. Scham, "weil ich mich wieder verletzt habe"; "versagt habe, anders mit dem Stress umzugehen") sowie eine weitere NSSV als Folge auf diese unangenehme Emotion (Abb. 1). Aus dieser kontingenten Abfolge aus (kurzfristiger) Erleichterung (negative Verstärkung) und Aktivierung selbstabwertender Gedanken kann man sich gut vorstellen, wie es bei unseren Patienten zu einem Teufelskreis mit wiederholten Selbstverletzungen (mitunter an nur einem Tag) kommen kann [Houben et al., 2017].
Insgesamt helfen diese Erkenntnisse aus der Verhaltensforschung in der Therapie von Patienten mit NSSV: Zum Beispiel kann in einer Verhaltensanalyse wie dem Stimulus-Organismus-Response-(Konsequenz)-Contingenz-(SORKC- oder SORC-) Modell [Kanfer und Saslow, 1965] dem Klienten im Sinne einer Psychoedukation verdeutlicht werden, wie die Selbstverletzung als negativer Verstärker wirkt und so die Wahrscheinlichkeit erhöht, dass man sich in der nächsten Stresssituation wieder verletzen wird. Da der erleichternde Effekt der NSSV beim Klienten oft so stark ist, sollte der Therapeut immer wieder verdeutlichen, dass es notwendig ist, Selbstverletzungen abzubauen und alternative Fertigkeiten zur Spannungsreduktion ("Skills" in der dialektisch-behavioralen Therapie) aufzubauen [Linehan, 1993]. Denn nur so kann die Kontingenz des Belohnungsreizes der NSSV aufgebrochen werden.

\section{Kann Suizidalität bei Borderline ähnlich verstanden werden wie NSSV?}

Brown et al. [2002] waren bei den ersten, die die Hintergründe von NSSV und SV miteinander verglichen haben. In beiden Fällen fanden sie, dass Emotionsregulation eine bedeutende Rolle spielt. Im Vergleich zu SV wurde NSSV allerdings häufiger eingesetzt, um bestimmte Gefühle herzustellen, sich selbst zu bestrafen, Ärger zum Ausdruck zu bringen oder sich abzulenken. SV hingegen wurden signifikant häufiger durchgeführt, damit es anderen besser geht. Tabelle 2 gibt nach dem aktuellen Stand der Literatur eine Übersicht zu den Gemeinsamkeiten und Unterschieden von NSSV und SV.

Nichtsdestotrotz sind SV nur ein Symptom von Suizidalität. Auch wenn Borderline-Patienten keine SV unter- 


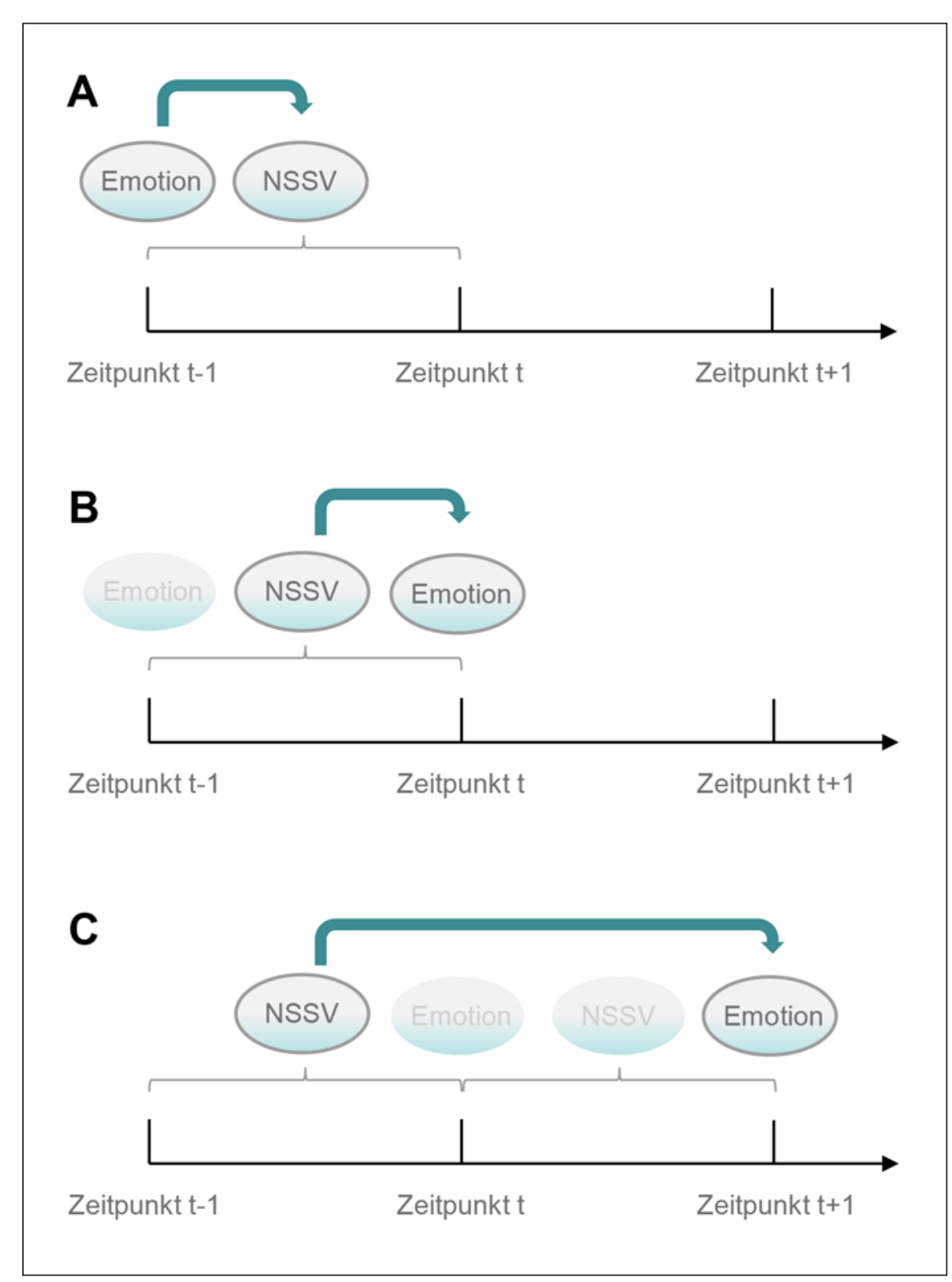

Abb. 1. Kontingenz von NSSV. A NSSV tritt bei BPS in der Folge auf eine (meist) aversive Emotion auf. B Auf das NSSV folgt (nach einer kurzfristigen Erleichterung) jedoch wiederum eine aversive Emotion (z.B. Scham, Selbsthass). C Die sich so einstellende, kontingente Abfolge von Emotion, NSSV und Emotion macht es möglich, mit einer hohen Wahrscheinlichkeit sogar die anschließende aversive Emotion nach einer nächsten NSSV vorhersagen zu können. Modifiziert nach Houben et al. [2017]. nehmen, leiden sie oft unter wiederkehrenden Suizidideationen, beschäftigen sich mit dem Tod und Suizid im Internet oder tauschen Suizidmethoden in sozialen Medien aus [Forsthoff et al., 2006]. Tatsächlich finden gehäufte NSSV und SV typischerweise in einer umschriebenen, relativ frühen Krankheitsphase der BPS statt [Zanarini et al., 2008], Suizidideationen hingegen sind häufig über viele Jahre vorhanden [Paris, 2019]. Nicht selten werden letztere als "nicht akute" Suizidalität auch im professionellen Umfeld abgetan. Brisanterweise kommt es aber dann tatsächlich bei diesen Patienten vor allem im späteren Krankheitsverlauf zu vollendeten Suiziden [Paris und Zweig-Frank, 2001]. D.h., Borderline-Patienten haben - statistisch gesehen - nicht ihr höchstes Suizidrisiko im jungen Alter und in der Krankheitsphase, wo sie wegen Krisen oder NSSV noch häufig in Notaufnahmen und geschützten Stationen behandelt werden, sondern eher nach Jahren erfolgloser Therapien in einem fortwährenden Zustand chronischer Suizidalität [Paris, 2019]. Es spricht daher Einiges dafür, neben dem klinischen Management von NSSV und SV, frühzeitig in der Psychotherapie wiederkehrende Suizidgedanken gezielt zu behandeln, um spätere Suizide zu verhindern. An dieser Stelle kommt jetzt die Frage auf, ob wir das bei NSSV hilfreiche Verstärkermodell auch auf chronische Suizidalität übertragen und ähnliche Konsequenz-Kontingenz-Abfolgen für das Auftreten von Suizidgedanken als Antwort auf aversive Gefühlszustände angenommen werden können. Mit anderen Worten: 


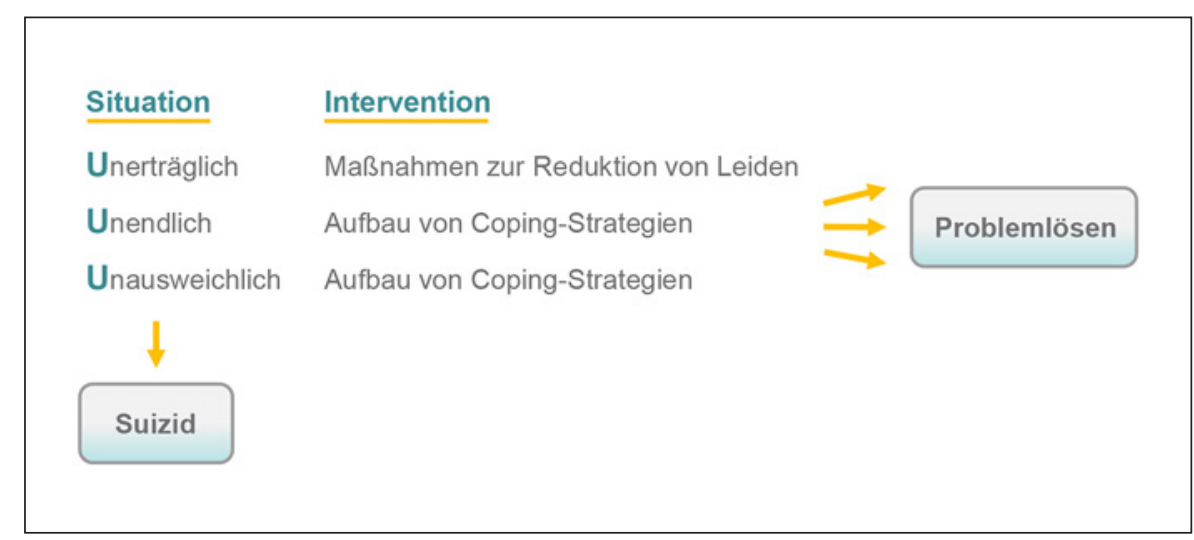

Abb. 2. Das Problemlöse-Modell der Suizidalität. In einer als unerträglich, unendlich oder unausweichlich erlebten Situation (englisch: "The Three Is": intolerable, interminable and inescapable) werden Problemlösestrategien benötigt, um das Leiden zu beenden. Theoretisch steht am Ende einer Kette von möglichen Maßnahmen, wenn sie erfolglos waren, immer auch der Suizid. Menschen mit wiederholten suizidalen Krisen sind überzeugt, dass Sui- zid eine Lösung jeglicher Problemsituationen darstellt und dass andere Strategien nicht zur Verfügung stehen oder nicht erfolgsversprechend sind. Als antisuizidale Intervention geht es nach diesem Modell einerseits darum, das Leid zu reduzieren (z.B. durch eine Medikation) und andererseits Fertigkeiten aufzubauen, mit einer Situation umzugehen oder sie zu verändern. Modifiziert nach Chiles und Strosahl [2004] und Bilsker und Forster [2003].

Tabelle 2. Funktionen von nichtsuizidaler Selbstverletzung und Suizidversuchen ${ }^{1}$

\begin{tabular}{|c|c|}
\hline Nichtsuizidale Selbstverletzung & Suizidversuch \\
\hline Regulation negativer Affekte, Emotionsregulation (häufigste & Emotionale Erleichterung, Nachlassen von psychischen \\
\hline Nennung (63-78\% in Taylor et al. [2018]) & $\begin{array}{l}\text { Schmerzen (von den meisten Patienten in Brown et al. [2002] } \\
\text { angegeben) }\end{array}$ \\
\hline Selbstbestrafung & $\begin{array}{l}\text { Interpersonelle Einflussnahme (wahrscheinlich weniger } \\
\text { bedeutsam als bei NSSV) }\end{array}$ \\
\hline Antidissoziativ (z.B. Schmerzreize stoppen Taubheitsgefühl) & $\begin{array}{l}\text { Damit es anderen besser geht/keine Last mehr für andere zu } \\
\text { sein (stärker als bei NSSV) }\end{array}$ \\
\hline $\begin{array}{l}\text { Interpersonelle Einflussnahme (z.B. Not/Leiden kommunizieren; } \\
\text { das Verhalten anderer beeinflussen; aktiv andere bestrafen/ } \\
\text { Schmerz zufügen; insgesamt weniger bedeutsam als } \\
\text { Affektregulation; } 33-56 \% \text { in Taylor et al. [2018]) }\end{array}$ & Gefühl von Kontrolle \\
\hline \multicolumn{2}{|l|}{ Antisuizidal (z.B. Stoppen von Suizidgedanken/-impulsen) } \\
\hline \multicolumn{2}{|l|}{ Sensationslust, Ablenkung ("etwas Aufregendes tun") } \\
\hline \multicolumn{2}{|l|}{$\begin{array}{l}\text { Zugehörigkeit (z.B. Teil einer Peer-Gruppe werden, mit anderen } \\
\text { ähnlich (nicht anders) sein) }\end{array}$} \\
\hline $\begin{array}{l}{ }^{1} \text { Bitte beachten Sie, dass die Daten [Tullis, 1998; Brown et al., } \\
\text { terschiedlichen Erhebungsformen mit unterschiedlichen Definitio } \\
\text { terviews und Fallberichte). Aus diesem Grund sind Faktoren, die } \\
\text { dargestellt. }\end{array}$ & $\begin{array}{l}\text {; Klonsky, 2007; Klonsky et al., 2013; Taylor et al., 2018] aus u } \\
\text { entstammen (validierte, strukturierte Fragebögen, klinische } \\
\text { hrscheinlich zusammenhängen oder ähnlich sind, in einer Ze }\end{array}$ \\
\hline
\end{tabular}

\section{Können Gedanken ähnlich wie ein Verhalten in ein Verstärkermodell gefasst werden?}

Wenn wir in der Psychotherapie mit Verhaltensanalysen arbeiten, fokussieren wir uns üblicherweise auf ein (meist) dysfunktionales Verhalten - also Dinge, die wir getan haben, die gewisse angenehme oder unangenehme Konsequenzen nach sich ziehen. Wie oben beschrieben, können diese Konsequenzen mich dahingehend verstärken, dass ich in einer vergleichbaren Situation nächstes Mal ähnlich handeln werde. Zum Beispiel, wenn ich in einer als ausweglos empfundenen Situation mit hoher Anspannung erfahren habe, dass Schneiden unmittelbar zu einer Erleichterung führt (negative Verstärkung) oder mir sogar das Gefühl gibt, ich habe mich/die Situation 
unter Kontrolle (positive Verstärkung). Ist es aber wirklich etwas anderes, wenn ich mich stattdessen gedanklich mit Suizid beschäftige, und ich dadurch eine Erleichterung erfahre, weil ich eine Vorstellung entwickle, dass ich aus der Situation herauskommen kann? Chiles und Strosahl [2004] haben beobachtet, dass Jugendliche, die durch Auslöser von innen (z.B. Denken an eigene Unzulänglichkeit) oder außen (z.B. durch Mobbing) starken seelischen Schmerz empfinden, Erleichterung erfahren, wenn sie in der Situation an Suizid denken. Kürzlich konnte in einer Echtzeit-Untersuchung über 28 Tage mit Smartphones (ähnlich wie bei der oben erwähnten Studie von Houben) dargestellt werden, dass bei Patienten mit einer Vorgeschichte von SV sich der Affekt immer dann verbesserte und Traurigkeit abnahm, wenn Suizidgedanken auftraten [Kleiman et al., 2018]. Die Autoren stellten daher die Frage, ob hier Suizidgedanken als Verstärker fungieren können. Ähnlich wie verschiedene Verhaltensmuster (wie z.B. NSSV, Essstörung, Substanzkonsum) im Sinne der von Hayes [2004] beschriebenen Erfahrungsvermeidung verstanden werden können (Verlassen, Vermeiden oder Ändern einer unangenehmen Erfahrung), kann die gedankliche Beschäftigung mit Suizid die Funktion haben, Emotionen zu unterdrücken und seelisches Leiden zu kontrollieren [Murrell et al., 2014]. In der Bezugsrahmentheorie [Hayes et al., 2001] und der Akzeptanz-Commitment-Therapie (ACT) [Hayes, 2004] geht Hayes davon aus, dass menschliches Verhalten immer eine Funktion hat. So kann auch die gedankliche Beschäftigung (eine Form von "Verhalten") mit Suizid als eine gelernte und verstärkte Art des Problemlösens betrachtet werden, z.B. indem intensive negative Emotionen abgeschaltet oder vermieden werden[Chiles und Strosahl, 2004] (Abb. 2). Nach Hayes ist die Erleichterung (relief) in solchen Situationen nicht direkt konditioniert (d.h., die Person hat nicht bereits erlebt, dass der Tod von seelischen Schmerzen befreit), wie es bei der klassischen Konditionierung der Fall ist (z.B. Vermeiden geschlossener Räume bei der Agoraphobie oder dem Nachlassen von Zwangsgedanken (z.B. Kontamination) beim Ausführen von Zwangshandlungen (z.B. exzessivem Händewaschen)). Stattdessen werden sogenannte verbale "wenn..., dann"-Assoziationen konstruiert (z.B.: "Wenn ich sterbe, werde ich nicht mehr von meinen Klassenkameraden gemobbt") [Hayes, 1992]. Dieses "verbale Verhalten" unterliegt Langzeit-Konditionierungsprozessen und kann aversive (negative) oder appetitive (positive) Konsequenzen beinhalten (zur Übersicht: Murrell et al. [2014]).

Wendet man nun dieses Modell auf die chronische Suizidalität bei der BPS an, können Suizidideationen (das "verbale Verhalten") Hoffnungslosigkeit, Hilflosigkeit und unerträgliche Wut reduzieren und gleichzeitig als ein negativer Verstärker wirken, der die Wahrscheinlichkeit für Suizidassoziationen in einer ähnlichen aversiven Situation erhöht (Abb. 3).

Der (negativ) verstärkende Effekt, den jemand durch die Erleichterung in einer solchen Situation erfährt, in dem er an den Tod denkt, mag sogar noch größer sein, wenn er sich genau vorstellt, wann, wo und wie er Suizid begeht [Chiles und Strosahl, 2004]. So kann es zu einer Gewohnheit werden, im Internet nach Suizidmethoden $\mathrm{zu}$ recherchieren, Suizid auf entsprechenden OnlinePlattformen zu diskutieren oder den Suizid vorzubereiten (z.B., indem Tabletten gesammelt, Orte zum Erhängen oder eine Brücke zum Springen ausgesucht werden). Jemand, der Suizidideationen hat, erfährt von dieser Perspektive aus die "ultimative Verstärkung" - einen Weg, der es ihm erlaubt, dauerhaft und vollständig schwierige emotionale Erfahrungen zu kontrollieren [Chiles und Strosahl, 2004].

\section{Süchtig machende und ansteckende Suizidalität?}

Tullis [1998] hat in seiner Theorie der Suizid-Abhängigkeit (A theory of suicide addiction, 1998) die hier erwähnten Verstärkermechanismen an seinen Patienten eindrucksvoll beschrieben: "Über Suizid nachzusinnen kann bei einigen Menschen durchaus angenehm sein, oder es stoppt zumindest psychisches Leiden. Suizidale Gedanken oder Verhalten kann eine Form der Selbstmedikation bei diesen Menschen sein; das erneute Durchleben früherer Suizidversuche in Gedanken oder die bildliche Vorstellung des eigenen Tods kann zu einem Ritual (oder gar Trance-ähnlichem Rauschzustand) werden, das ein Gefühl von Kontrolle und einen möglichen Ausweg aus dem Leiden vermittelt." Tullis selbst beobachtete bei seinen Patienten diesen Beruhigungseffekt von Suizidgedanken, der bis hin zu rauschähnlichen oder euphorischen Zuständen reichte (rush, high, thrill, exhilaration). Er stellte auch einen Toleranzeffekt dieser Wirkung über die Zeit fest, so dass Patienten - ähnlich wie es bei Substanzabhängigkeiten beobachtet wird - zwangsähnliche Rituale und Verhaltensmuster entwickelten, wenn sie z.B. Tabletten oder allerhand Paraphernalien für den Suizid sammeln und horten. Tullis Beobachtungen unterstützen also die Hypothese, dass bei manchen Menschen die Beschäftigung mit Suizidgedanken angenehm ist, seelische Schmerzen reduziert und eine Art "Way of life" werden kann [Paris, 2004] - psychologisch gesprochen: eine Beschäftigung, die Verstärker- und Kontingenzmechanismen unterliegt.

Es wird (z.B. in akut-psychiatrischen Stationen) nicht selten beobachtet, dass Borderline-Patienten dysfunktionale Verhaltensweisen von anderen Borderline-Mitpatienten "abgucken" und übernehmen. So fängt z.B. eine Patientin an, sich zu ritzen, obwohl sie es nie zuvor getan hatte, was wiederum nahelegt, dass es (zumindest kurzfristig) eine deutlich positive Konsequenz beinhaltet: He- 


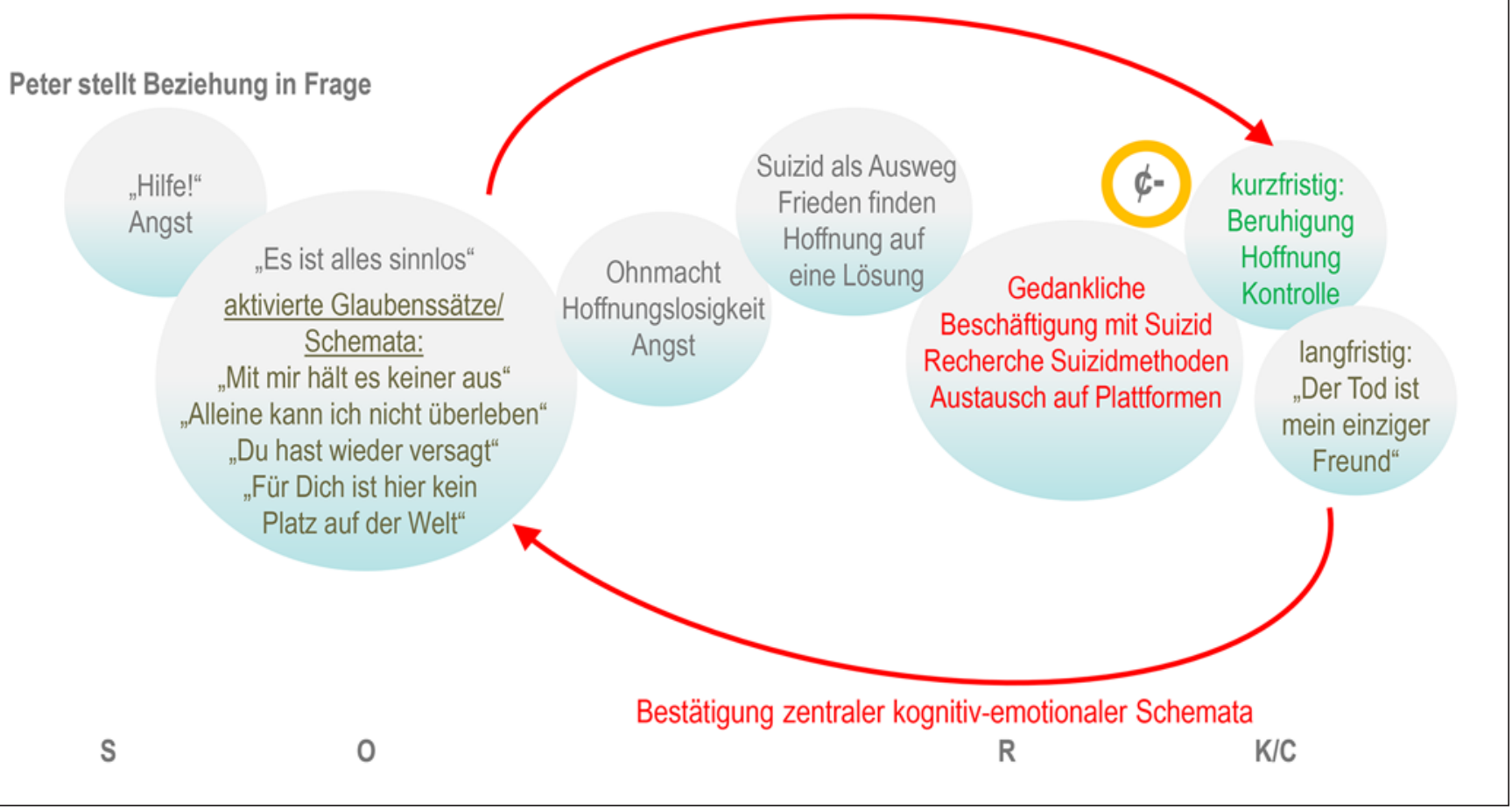

Abb. 3. Kettenanalyse und SORKC-Modell von Suizidalität. Jessicas Verhaltens-(Ketten-) Analyse von suizidalen Symptomen (hier: Suizidgedanken, Internetrecherche, Suizidvorbereitung), die auftraten, nachdem ihr Freund die Beziehung in Frage gestellt hat (Auslöser, S). Jessica ist zunächst perplex und hat Angst, Peter zu verlieren (primäre Emotionen; Traurigkeit wäre hier z.B. auch denkbar). Die ersten Gefühle verschwinden rasch, nachdem Jessicas maladaptive kognitive Schemata $(\mathrm{O})$ aktiviert sind (ihre Interpretation der Situation vor dem Hintergrund ihrer Kindheitserfahrungen). Diese Bewertungen wiederum aktivieren sogenannte sekundäre Emotionen (wie z.B. Hilflosigkeit, Hoffnungslosigkeit, Panik) und verursachen zusätzliches seelisches Leid und Anspannung. Es sei darauf hingewiesen, dass der Übergang von primären zu sekundären Emotionen vor allem bei BPS sehr schnell gehen kann. Oft sogar so schnell, dass die primäre Emotion überhaupt nicht wahrgenommen wird [Linehan, 1993]. Gedanken an den eigenen Suizid, der Austausch mit Peers oder im Chat auf entspre-

rausfinden, was im Moment am besten hilft, Spannung zu reduzieren; mit Peers verbunden fühlen, die mich verstehen [Taiminen et al., 1998; Prinstein et al., 2010]. Jetzt können wir spekulieren, dass der Austausch von Suizidthemen über soziale Medien ein ähnliches Phänomen darstellt: Patienten “testen” verschiedene Suizidassoziationen an, während sie untereinander darüber diskutieren und erfahren in dieser Konditionierung eine (erstmalige) Verstärkung.

\section{Bewertungen und Glaubenssätze sind die toxischen}

\section{Zutaten des Verstärkermodells der Suizidalität}

Wenn wir nach einem geeigneten Ansatz suchen, Suizidalität bei BPS anzugehen, wird die Auseinandersetzung mit den Hintergründen und Motiven sprichwört- chenden Internetseiten beruhigt Jessica und lässt in ihr ein Gefühl von Hoffnung und Kontrolle entstehen ("Ich kann da immer noch rauskommen", "Es gibt einen Ausweg", "Ich muss nicht weiter leiden"). Die Kontingenz zwischen psychischem Schmerz und der Erleichterung $(\mathrm{K} / \mathrm{C})$ wirkt als ein negativer Verstärker ( $\$-)$, der die Wahrscheinlichkeit erhöht, dass in der nächsten Anspannungssituation ebenfalls Suizidalität auftritt. Andererseits folgen langfristig (beinhaltet hier alles direkt nach "kurzfristig") auch aversive Emotionen wie Insuffizienzgefühle, Scham, Einsamkeit ("Ich gehöre einfach nicht in diese Welt", "Ich bin beziehungsunfähig", "Ich bin alleine, der Tod ist mein einziger Freund"), die wiederum die dysfunktionalen Grundannahmen bestärken und bestätigen. So entsteht ein Teufelskreis aus der Verstärkung der Suizidalität und der wiederholten Bestätigung zentraler kognitiv-emotionaler Schemata, die letztendlich zu wiederkehrender, anhaltender und schließlich chronischer Suizidalität führen.

lich existenziell. In der Sprache der Verhaltensanalyse bestimmt die Organismus-Variable, wie wir einen Stimulus verarbeiten und letztendlich darauf reagieren [Ellis, 1969]. Neben rein biologischen Faktoren (z.B. Wachheit, genetische Disposition, andere körperliche Einflussfaktoren) ist die Organismus-Variable wesentlich durch unsere eigenen Erfahrungen beeinflusst - Botschaften, die wir als Kind erhalten haben, oder Verhaltensmodelle, die wir vor uns hatten [Young et al., 2003]. Ähnlich wie in dem klinischen Beispiel in Abbildung 1 haben suizidale Jugendliche und Borderline-Patienten nach ihren frühen Erfahrungen von Invalidierung oder Traumatisierung häufig Vorstellungen, wertlos, unangemessen, zurückgewiesen oder schuldig zu sein [Young et al., 2003; Murrell et al., 2014]. Typische Beispiele hierfür sind Gedanken, 
wie "Ich kann nicht alleine leben", "Ich bin falsch", "Ich habe keinen Platz in der Welt", "Ich bin ein schlechter Mensch", "Das schaffe ich nicht". Diese automatischen Gedanken sind sehr robuste Überzeugungen von sich und der Welt um einen herum ("Glaubenssätze" in der dialektisch-behavioralen Therapie), die durch Signale von innen oder außen aktiviert (bewusst) werden [Beck, 1976], z.B., jedes Mal, wenn man sich beleidigt, enttäuscht, zurückgewiesen, einsam (...) fühlt. Es konnte kürzlich gezeigt werden, dass aversive Gefühlszustände in Abhängigkeit der Schwere der BPS hoch kontingent mit spezifischen dysfunktionalen Verhaltensmustern verknüpft werden (z.B. heftige Wut nach Beleidigung, NSSV nach Enttäuschung) [Miskewicz et al., 2015].

Mit Hilfe der Verhaltensanalyse kann der Therapeut nun diese Verstärkerkontingenzen innerhalb des Teufelskreises aus Schlüsselreizen, aktivierten Glaubenssätzen und darauffolgenden Suizidgedanken aufdecken. Er kann nun adaptivere Verhaltensalternativen erarbeiten und Hindernisse (z.B. intensive Gefühle, Angst, Scham, Schuld oder falsche Annahmen wie "Ich bin ein Versager", "Ich habe kein Recht dazu") identifizieren, die die Anwendung funktionalen Verhaltens verhindern [Linehan, 1993].

Ähnlich wie im Verstärkermodell von NSSV können wir annehmen, dass die Verstärkerfunktion von Suizidgedanken und -handlungen zentrale Grundannahmen bestätigt und so das dysfunktionale System der chronischen Suizidalität stabilisiert und aufrechterhält. In diesem Punkt der langfristigen Dynamik verhält es sich bei NSSV anders: Das schnelle Auftreten einer erneuten aversiven Emotion als Antwort auf das NSSV wie oben beschrieben [Houben et al., 2017] kann hier als Bestrafung im behavioralen Sinne wirken und trägt möglicherweise dazu bei, dass von dem selbstverletzenden Verhalten mit der Zeit abgelassen wird (eine häufige klinische Beobachtung im Verlauf der BPS). Bei der chronischen Suizidalität fehlt dieser ungewünschte Effekt, so dass sie oft über viele Jahre bestehen bleibt.

\section{Therapeutische Interventionen, die aus dem}

Verstärkermodell der Suizidalität abgeleitet werden

können

Die situationsspezifischen Motive und psychischen Funktionen hinter suizidalem Verhalten anzusprechen mag ein erster, aber sehr wirkungsvoller Schritt in der therapeutischen Arbeit mit suizidalen Borderline-Klienten sein. Der Klient kann eine substantielle Validierung erfahren, indem er seine eigene Verhaltensanalyse mit dem Therapeuten bespricht und beide Seiten die aufrechterhaltenden Verstärkermechanismen verstehen (Abb. 3). Die Normalisierung von Suizidgedanken und -verhalten aus der Perspektive des Betroffenen ("Wenn ich in dieser Situation gewesen wäre, hätte ich ähnlich gehandelt/gefühlt") und im Vergleich zu anderen ("Viele in deinem Alter haben ernst- hafte Gedanken, sich das Leben zu nehmen - das ist nicht so ungewöhnlich oder komisch") kann Scham (mit den Anforderungen des Lebens nicht zurecht zu kommen) abbauen [Murrell et al., 2014]. In dem so geschaffenen von Akzeptanz getragenen Behandlungsrahmen kann Suizidalität aufrichtig und ehrlich diskutiert werden.

Die Arbeit mit dysfunktionalen Grundannahmen und Überzeugungen ist wahrscheinlich einer der herausforderndsten aber langfristig unausweichlichen Schritte in der Psychotherapie der chronischen Suizidalität. Um an dieser Stelle eine kognitiv-emotionale Neubewertung zu erreichen, kommen sowohl kognitive als auch emotionale Expositionstechniken zum Einsatz [Linehan, 1993; Young et al., 2003]. Auch können sogenannte DefusionsTechniken der ACT (Distanzierungs- und Trennungstechniken von Gedanken und Gefühlen) negative Urteile und Glaubenssätze abschwächen [Murrell et al., 2014].

Vor dem Hintergrund hoher Raten von Traumatisierung und tiefgreifender Invalidierungserfahrungen bei chronisch suizidalen Patienten hat insbesondere die expositionsbasierte Traumatherapie einen hohen Stellenwert in der Behandlung suizidaler Symptome. Wie die neusten Entwicklungen bei der dialektisch-behavioralen Therapie für die posttraumatische Belastungsstörung zeigen, kann (und wahrscheinlich sollte) diese Konfrontation frühzeitig im Rahmen eines spezialisierten Behandlungsrahmens erfolgen [Bohus et al., 2013, 2019]. Aus der ACT und der mitgefühlsorientierten Therapie (compassion-focussed therapy) nach Gilbert kommen Techniken, die den anschließend notwendigen Trauerprozess und schließlich die Akzeptanz dessen, was in der Vergangenheit passiert ist, unterstützen [Hayes, 2004; Gilbert, 2010].

Parallel ist es wichtig, alternative nicht suizidale Verhaltensweisen aufzubauen, die zumindest anfänglich durch den Therapeuten verstärkt werden. Langfristig ist das Ziel, ein natürliches Verstärkersystem einzurichten, z.B. indem die Werte des Patienten genutzt werden: Aufbau und Zugehörigkeit zu einem Freundeskreis, Gefühl von Verbundensein, indem man sich sozial integriert und Verantwortung übernimmt (ehrenamtliche Tätigkeit, sich ein Haustier halten, ...). Werteorientierung oder werteorientiertes Handeln kann man als eine Form von Hayes "verbalem Verhalten" auffassen und davon ausgehen, dass es ebenso langfristig konditioniert werden kann: "Wenn ich lebe/am Leben bleibe, werden meine Eltern vielleicht sehen, wie ich eines Tages einen Schulabschluss erreiche" oder "Wenn ich Suizid begehe, wird es meinen Eltern sehr weh tun, zu meiner Beerdigung zu gehen" [Murrell et al., 2014].

Ohne Anspruch auf Vollständigkeit soll die Tabelle im Anhang eine Übersicht über mögliche Interventionen geben, die aus einem individuellen Verstärkermodell der Suizidalität abgeleitet werden können (online suppl. Zu- 
Tabelle 3. Therapeutische Begriffe

\begin{tabular}{|c|c|}
\hline ACT & Akzeptanz-Commitment-Therapie \\
\hline CFT & Mitgefühlsorientierte Therapie (compassion-focussed therapy) \\
\hline Commitment & $\begin{array}{l}\text { Bereitschaft; Umfassendes Einlassen auf die Therapie/einen therapeutischen Schritt. Geht über } \\
\text { Therapiemotivation hinaus }\end{array}$ \\
\hline Der neue Weg & $\begin{array}{l}\text { Werte- und zielorientierter Weg unter Einsatz funktionaler Strategien zur Problembewältigung (in } \\
\text { Abgrenzung zum "alten Weg") }\end{array}$ \\
\hline DBT & Dialektisch-behaviorale Therapie \\
\hline Glaubenssatz & Automatische Gedanken, dysfunktionale Überzeugungen von sich und der Welt \\
\hline Hochstress & $\begin{array}{l}\text { Psychische Anspannung, die in der DBT im Bereich von } 70-100 \% \text { definiert wird; typische Symptome sind: } \\
\text { Tunnelblick, Gedankenkreisen, starke muskuläre Anspannung, verminderter Realitätsbezug bis hin zur } \\
\text { Dissoziation }\end{array}$ \\
\hline Kettenanalyse & $\begin{array}{l}\text { Form der Verhaltensanalyse, bei der Emotionen, Kognitionen und sichtbares Verhalten in } \\
\text { aufeinanderfolgenden Kettenelementen dargestellt werden }\end{array}$ \\
\hline Primäre Emotion & $\begin{array}{l}\text { Emotion, die direkt mit der Situation zu tun hat und in der Regel auch als erste Emotion wahrgenommen } \\
\text { wird; bei BPS kann sie vor dem Hintergrund schwieriger Erfahrungen sehr kurz oder gar nicht } \\
\text { wahrgenommen werden, da rasch oder sofort sekundäre Emotionen präsent sind }\end{array}$ \\
\hline Sekundäre Emotion & $\begin{array}{l}\text { Emotion, die nach Bewertung/Interpretation eines Auslösers auftritt; im Gegensatz zur primären Emotion } \\
\text { kann sie keinen Bezug zur aktuellen Situation aufweisen oder aber unangemessen stark sein }\end{array}$ \\
\hline Skill & $\begin{array}{l}\text { Fertigkeit. Im Sinne der DBT eine alltagstaugliche, selbstinstruierte Selbstregulation (auf mentaler oder } \\
\text { Handlungsebene), die weder für mich noch andere kurz- oder langfristig schädlich ist - oder zumindest } \\
\text { deutlich weniger schädlich als das ursprüngliche Problemverhalten (z.B. Riechen an Ammoniak statt } \\
\text { Selbstverletzung) }\end{array}$ \\
\hline Telefoncoaching & $\begin{array}{l}\text { Form der therapeutischen Unterstützung in Krisensituationen; der Therapeut coacht den Klienten am } \\
\text { Telefon während einer Krise }\end{array}$ \\
\hline Validierung & $\begin{array}{l}\text { Durch Mimik, Gestik, Körperhaltung oder Sprache verdeutlichen, dass man die subjektive Sicht des } \\
\text { Gegenübers für stimmig und nachvollziehbar hält; wiederholte und intensive Erlebnisse von Invalidierungen } \\
\text { ("Du spinnst doch”, "Kannst du nicht einfach so sein wie ein normales Kind”, "Warum weinst du schon } \\
\text { wieder", Gewalterfahrungen, Mobbing ...) spielen im Genesemodell der Borderline-Störung eine zentrale } \\
\text { Rolle }\end{array}$ \\
\hline
\end{tabular}

satztab. 1; für das ganze online suppl. Material, siehe www.karger.com/doi/10.1159/000518239). Es handelt sich hierbei um Standardtechniken der Verhaltenstherapie, wie sie auch in der dialektisch-behavioralen Therapie, ACT oder der mitgefühlsorientierten Therapie zur Anwendung kommen: Validierungstechniken, Psychoedukation, kognitive Techniken, Techniken der Emotionsregulation und -exposition, Aufbau alternativer Verhaltensweisen und Fertigkeiten (therapeutische Begriffe in Tab. 3). Sie beziehen sich auf das klinische Beispiel in Abbildung 1, können aber auch auf andere Klienten mit chronischer Suizidalität übertragen werden.

\section{Diskussion}

Ähnlich wie bei der NSSV können suizidale Gedanken und Verhaltensweisen verschiedene Hintergründe und Funktionen haben. Im Wesentlichen führen sie bei den Betroffenen dazu, dass seelische Schmerzen abnehmen, sei es durch die Reduktion aversiver Spannung, sei es, indem sie einen möglichen Ausweg aufzeigen, oder indem sie ein Gefühl von Kontrolle über schwierige Gefühle wie Schuld und Scham geben. Es ist also gut nachvollziehbar, dass Verstärkermechanismen auch bei der chronischen Suizidalität eine Rolle spielen. Tatsächlich konnte dieses Konzept in einer kürzlich erschienen Studie neurobiologisch erhärtet werden: Mittels autobiografischer Transkripte und Imaginationen wurden frühere suizidale Episoden während einer funktionellen Kernspin-Tomografie wiedererlebt. Hierbei zeigte sich, dass der seelische Schmerz, der suizidales Verhalten getriggert hatte, mit einer Abnahme präfrontaler Hirnaktivität verbunden war, das Planen und Ausführen von suizidalen Impulsen (in der gedanklichen Vorstellung) als Antwort auf den seelischen Schmerz aber mit einer Zunahme der Hirnaktivität im medialen präfrontalen Kortex, dem anterioren Cingulum sowie dem Hippocampus - 
was nach Einschätzung der Autoren nahelegt, dass zielgerichtetes suizidales Verhalten (in der Vorstellung) zu einer Abnahme von seelischem Schmerz führt [Reisch et al., 2010].

Immer noch besteht eine große Lücke in der Literatur, was die psychologischen Funktionen der einzelnen suizidalen Symptome betrifft. Lebensüberdruss, passive Todeswünsche, die gedankliche Vorstellung des eigenen Todes oder die Durchführung eines SV können möglicherweise sehr unterschiedliche Funktionen und neurobiologische Effekte haben - und zwar zwischen den Betroffenen, aber auch innerhalb einer Person in Abhängigkeit der Situation. In einer vergleichenden Untersuchung der Hypothalamus-Hypophysen-Nebennieren (HPA)Achse bei depressiven Patienten zeigte sich z.B., dass nach einem Suizidversuch die Stresshormon-Antwort stark herunterreguliert ist im Vergleich zu Patienten, die "nur" Suizidgedanken hatten [Pfennig et al., 2005]. In einer prospektiven Studie über 18 Monate war induzierter Peer-Stress prädiktiv für das Auftreten von Suizidgedanken unabhängig von der HPA-Achsen-Regulation, für das Auftreten eines Suizidversuches jedoch nur bei Patienten mit einer herunterregulierten HPA-Achse [Eisenlohr-Moul et al., 2018]. Epigenetische Mechanismen scheinen bei der Pathophysiologie der Suizidalität, insbesondere auch der Regulation der HPA-Achse, eine Rolle zu spielen [Roy und Dwivedi, 2017]. Sie könnten einige zeitlich überdauernde Effekte (z.B. die Hyporeaktivität der HPA-Achse), wie sie bei wiederholten Suizidversuchen beobachtet werden, erklären. Die Bedeutung dieser Befunde und deren Zuordnung in psychologische Modelle der Suizidalität müssen sicher weiter erforscht werden. Die Herausforderung hierbei wird sein, anspruchsvolle neurobiologische und psychologische Untersuchungsmethoden miteinander zu kombinieren und eine klare Abgrenzung einzelner suizidaler Symptome vorzunehmen.

Auch aus der klinischen Sicht ist eine genaue Erhebung des Schweregrades der Suizidalität anhand der linearen Symptomatologie (Lebensüberdruß, passive Todeswünsche, handlungsweisende Suizidideationen und suizidvorbereitende Handlungen sowie Suizidversuch) wichtig, um eine zuverlässige Risikoeinschätzung für die Suizidgefahr beim Patienten vorzunehmen. Der Therapeut möchte an dieser Stelle auch wissen, welche Faktoren den Patienten von einem Suizidideator zu einem Suizidversucher werden lassen. In verschiedenen Modellen zur Suizidalität kommen Furchtlosigkeit vor dem Tod und Schmerztoleranz bei dieser Frage eine besondere Bedeutung zu [Joiner, 2005; O'Connor, 2011; Klonsky et al., 2013; Klonsky und May, 2015; Franklin et al., 2017]. Sehr wahrscheinlich stehen die robustesten Prädiktoren für einen späteren SV mit diesen beiden Faktoren in engem Zusammenhang, weil sie die Tatsache widerspiegeln, früher bereits den Verlust physischer Integrität und Unverletztheit erfahren zu haben (z.B. NSSV, früherer SV, Kindesmissbrauch). Auf der anderen Seite steht das Gefühl von Verbundensein als eines der stärksten suizidprotektiven Faktoren [Klonsky und May, 2015]. Nichtsdestotrotz mag die Zuverlässigkeit selbst der besten Prädiktoren (d.h. der negativ-prädiktive Wert in diesem Fall) zu gering sein, um es dem Therapeuten zu erlauben, einen Suizid auszuschließen - mit anderen Worten: Der klinische Nutzen von Prädiktoren in der konkreten Situation, wo es um eine Risikoeinschätzung geht, ist beschränkt [Burke et al., 2018; Paashaus et al., 2019; Ren et al., 2019]. Und es bleibt natürlich die Frage, was man dann mit einem Borderline-Patienten in der Psychotherapie macht, der Suizidrisikofaktoren trägt? Wie soll der Therapeut anhand dieser Informationen vorgehen, was soll er unterlassen? Ohne Zweifel sollte die chronische Suizidalität gerade bei diesen Patienten psychotherapeutisch angegangen werden. Nach einem allgemeinen Verständnis in der dritten Welle der Verhaltenstherapie kann unser Gehirn ein neues Verhalten nur lernen, bzw. eine korrigierende Erfahrung machen, wenn wir uns in derselben oder einer ähnlichen Situation befinden, die sonst das alte (dysfunktionale) Verhalten ausgelöst hat (diskutiert bei Young et al. [2003]). Mit anderen Worten muss der Patient in einer Situation alternativ handeln (oder denken), in der er bisher suizidal wurde. An dieser Stelle würde er nun wahrscheinlich keine neuen Erfahrungen machen, wenn er in ein Krankenhaus eingewiesen und somit die ambulante Therapie unterbrochen würde. Es würden vielmehr alte Grundannahmen bestätigt werden ("Ich habe wieder versagt", "Ich bekomme es alleine einfach nicht hin", "Ich gehöre bestraft, weil ich mich schlecht verhalte", "Suizid ist noch die beste Option"). Im Gegenteil sollte der Therapeut dem Patienten gerade in dieser Zeit aktiv zur Seite stehen. Das könnte z.B. in einer Krise oder in einem Zustand aktivierter kritischer Emotionen (von denen der Therapeut weiß, dass sie mit suizidalem Verhalten bei dem Patienten verknüpft sind) so aussehen, dass der Therapeut den Patienten anleitet, seine Emotionen zu regulieren, mit ihm die Situation reflektiert (Metaperspektive) und ihn konkret unterstützt, alternatives Verhalten anzuwenden. Telefon- und Online-Coaching, Techniken der Emotionsregulation und Stresstoleranz sowie eine eingehende Verhaltensanalyse von suizidalem Verhalten und Denken sind therapeutische Werkzeuge, die hier hilfreich sein können.

Die intensive Arbeit an der Seite suizidaler Patienten ist oft herausfordernd und braucht auch für den Therapeuten einen sicheren Rahmen. Neben einem Commitment auf beiden Seiten ("den neuen Weg zu gehen", "einen Weg zu finden, am Leben zu bleiben", "bereit zu sein für die Konfrontation") werden folgende Bedingungen 
für eine Psychotherapie der chronischen Suizidalität bei Borderline empfohlen [Linehan, 1993]: Ein Non-Suizidvertrag mit möglichst konkreten und individuell zutreffenden Formulierungen ("Ich werde keinen Suizidversuch unternehmen", "Ich werde keine Tabletten für einen Suizid sammeln", "Ich lege Suizidpläne gegenüber meinem Therapeuten offen"...), ein funktionierender und eingeübter (!) Krisenplan (Skillskette mit Hochstress-Skills, Fertigkeiten zur Emotionsregulation und Krisenkontakten mit Prioritätenreihenfolge) sowie klare Kontingenzvereinbarungen (was passiert nach NSSV, einem Suizidversuch, die Therapie schädigendem Verhalten, ...).

Es besteht weiterhin ein großer Bedarf, validierte antisuizidale Interventionen einer größeren Zahl Betroffener zugänglich zu machen. Neuere Suizidpräventionsprogramme über das Internet und soziale Medien sollen niederschwellig Risikopopulationen erreichen und das Wissen über Suizidprävention verbreiten [Wasserman et al., 2015; Robinson et al., 2018]. Es braucht in der Therapie aber oft Zeit, eine vertrauensvolle Therapeut-PatientenBeziehung sowie eine nichtwertende Haltung des Therapeuten, um den individuellen Hintergrund von Suizidalität zu verstehen und Funktionalitäten herauszuarbeiten. Das hier vorgestellte Verstärkermodell der Suizidalität wendet klassische verhaltenstherapeutische Techniken bei chronisch suizidalen Borderline-Patienten an und ist insofern nicht neu. Es integriert theoretische Konzepte, die sich bereits für das Verständnis verwandter Phänomene wie NSSV oder SV als hilfreich erwiesen haben. Es beinhaltet bekannte Interventionen, die in der Behandlung von Suizidalität und der BPS wirkungsvoll sind, wie z.B. die dialektisch-behaviorale Therapie und ACT. Besonders (und insofern neu) ist in diesem Modell jedoch die Betonung von Verstärkermechanismen für die Entstehung und Aufrechterhaltung von chronischer Suizidalität. Es unterstützt uns dabei, mit dem Patienten ein ge- meinsames Commitment zu finden, gezielte therapeutische Interventionen $\mathrm{zu}$ planen, die die bisherigen suizidalen Kontingenzen hilft aufzulösen.

\section{Hinweis}

Aus Gründen der besseren Lesbarkeit wird auf die gleichzeitige Verwendung der Sprachformen männlich, weiblich und divers $(\mathrm{m} / \mathrm{w} / \mathrm{d})$ verzichtet. Sämtliche Personenbezeichnungen gelten gleichermaßen für alle Geschlechter.

\section{Acknowledgement}

Der Autor dankt Frau Cordula Leutenbauer und Frau Julia Mayer für den hervorragenden fachlichen Austausch.

\section{Statement of Ethics}

Bei den im Artikel berichteten klinischen Beispielen handelt es sich um typische, jedoch fiktive Fälle. Etwaige Gemeinsamkeiten mit real existierenden Personen sind rein zufällig.

\section{Conflict of Interest Statement}

Der Autor erklärt hiermit, dass kein Interessenkonflikt besteht.

\section{Funding Sources}

Es besteht keine finanzielle Unterstützung für die Erstellung oder Veröffentlichung des Manuskripts.

\section{Author Contributions}

Der Autor bestätigt, der alleinige Autor der Arbeit zu sein und einer Veröffentlichung zugestimmt zu haben.

\section{Literatur}

American Psychiatric Association. Diagnostic and statistical manual of mental disorders [Internet]. 5th ed. Washington: American Psychiatric Association; 2013. https://doi. org/10.1176/appi.books.9780890425596.

Beck AT. Cognitive therapy and the emotional disorders. New York: International Universities Press; 1976.

Bilsker D, Forster P. Problem-solving intervention for suicidal crises in the psychiatric emergency service. Crisis. 2003;24(3):134-6.

Bohus M, Dyer AS, Priebe K, Krüger A, Kleindienst N, Schmahl C, et al. Dialectical behaviour therapy for post-traumatic stress disorder after childhood sexual abuse in patients with and without borderline personality disorder: a randomised controlled trial. Psychother Psychosom. 2013;82(4):221-33.
Bohus M, Schmahl C, Fydrich T, Steil R, MüllerEngelmann M, Herzog J, et al. A research programme to evaluate DBT-PTSD, a modular treatment approach for Complex PTSD after childhood abuse. Borderline Personal Disord Emot Dysregul. 2019 Mar;6(1):7.

Brown MZ, Comtois KA, Linehan MM. Reasons for suicide attempts and nonsuicidal self-injury in women with borderline personality disorder. J Abnorm Psychol. 2002 Feb;111(1): 198-202.

Burke TA, Ammerman BA, Knorr AC, Alloy LB, McCloskey MS. Measuring Acquired Capability for Suicide within an Ideation-to-Action Framework. Psychol Violence. 2018 Mar;8(2):277-86.

Chiles JA, Strosahl KD. Clinical manual for assessment and treatment of suicidal patients
[Internet]. Washington: American Psychiatric Publishing, 2004 [cited 2019 Dec 1]. Available from: https://www.scribd.com/ document/345714964/John-A-Chiles-KirkD-Strosahl-Clinical-Manual-for-Assessment-and-Treatment-of-Suicidal-PatientsAmerican-Psychiatric-Publishing-Inc2004-pdf.

Curtin SC, Warner M, Hedegaard H. Increase in suicide in the United States, 1999-2014. NCHS Data Brief. 2016 Apr;241:1-8.

Eisenlohr-Moul TA, Miller AB, Giletta M, Hastings PD, Rudolph KD, Nock MK, et al. HPA axis response and psychosocial stress as interactive predictors of suicidal ideation and behavior in adolescent females: a multilevel diathesis-stress framework. Neuropsychopharmacology. 2018 Dec;43(13):2564-71. 
Ellis A. A cognitive approach to behavior therapy. Int J Psychiatry. 1969 Dec;8(6):896-900.

Forsthoff A, Hummel B, Möller HJ, Grunze H. [Suicidality and the Internet. Danger from new media]. Nervenarzt.2006 Mar;77(3):343-

Franklin JC, Ribeiro JD, Fox KR, Bentley KH, Kleiman EM, Huang X, et al. Risk factors for suicidal thoughts and behaviors: a meta-analysis of 50 years of research. Psychol Bull. 2017 Feb;143(2):187-232.

Gilbert P. Compassion focused therapy: distinctive features. London: Routledge; 2010. https://doi.org/10.4324/9780203851197.

Hayes SC. Acceptance and commitment therapy, relational frame theory, and the third wave of behavioral and cognitive therapies. Behav Ther. 2004;35(4):639-65.

Hayes SC. Verbal relations, time, and suicide [Internet]. In: Hayes SC, Hayes LJ, editors. Understanding verbal relations. Reno: Context Press; 1992. p. 109-18 [cited 2019 Dec 1]

Hayes SC, Barnes-Holmes D, Roche B, editors. Relational frame theory: a post-Skinnerian account of human language and cognition [Internet]. New York: Springer US; 2001. https://doi.org/10.1007/b108413.

Houben M, Claes L, Vansteelandt K, Berens A, Sleuwaegen E, Kuppens P. The emotion regulation function of nonsuicidal self-injury: a momentary assessment study in inpatients with borderline personality disorder features. J Abnorm Psychol. 2017 Jan;126(1):89-95.

Joiner TE. Why people die by suicide. Cambridge: Harvard University Press; 2005.

Kanfer FH, Saslow G. Behavioral analysis: an alternative to diagnostic classification. Arch Gen Psychiatry. 1965 Jun;12(6):529-38.

Kirtley OJ, O'Carroll RE, O'Connor RC. The role of endogenous opioids in non-suicidal selfinjurious behavior: methodological challenges. Neurosci Biobehav Rev. 2015 Jan;48:186-

Kleiman EM, Turner BJ, Fedor S, Beale EE, Picard RW, Huffman JC, et al. Digital phenotyping of suicidal thoughts. Depress Anxiety. 2018 Jul;35(7):601-8.

Klonsky ED. The functions of deliberate self-injury: a review of the evidence. Clin Psychol Rev. 2007 Mar;27(2):226-39.

Klonsky ED, May AM. The three-step theory (3ST): a new theory of suicide rooted in the "ideation-to-action" framework. Int J Cogn Ther. 2015;8(2):114-29.

Klonsky ED, May AM, Glenn CR. The relationship between nonsuicidal self-injury and attempted suicide: converging evidence from four samples. J Abnorm Psychol. 2013 Feb; 122(1):231-7.

Klonsky ED, May AM, Saffer BY. Suicide, suicide attempts, and suicidal ideation. Annu Rev Clin Psychol. 2016;12(1):307-30.

Lieb K, Zanarini MC, Schmahl C, Linehan MM, Bohus M. Borderline personality disorder. Lancet. 2004 Jul;364(9432):453-61.

Linehan MM. Suicidal people. One population or two? Ann N Y Acad Sci. 1986;487 1 Psychobiology:16-33.
Linehan MM. Cognitive-behavioral treatment of borderline personality disorder. New York: Guilford Publications; 1993.

Linehan MM, Comtois KA, Brown MZ, Heard HL, Wagner A. Suicide Attempt Self-Injury Interview (SASII): development, reliability, and validity of a scale to assess suicide attempts and intentional self-injury. Psychol Assess. 2006 Sep;18(3):303-12.

Linehan MM, Comtois KA, Ward-Ciesielski EF. Assessing and managing risk with suicidal individuals. CognitBehavPract.2012;19(2):21832 .

Linehan MM, Goodstein JL, Nielsen SL, Chiles JA. Reasons for staying alive when you are thinking of killing yourself: the reasons for living inventory. J Consult Clin Psychol. 1983 Apr;51(2):276-86

Miller AB, Eisenlohr-Moul T, Giletta M, Hastings PD, Rudolph KD, Nock MK, et al. A withinperson approach to risk for suicidal ideation and suicidal behavior: examining the roles of depression, stress, and abuse exposure. J Consult Clin Psychol. 2017 Jul;85(7):712-22.

Miskewicz K, Fleeson W, Arnold EM, Law MK, Mneimne M, Furr RM. A Contingency-Oriented Approach to Understanding Borderline Personality Disorder: Situational Triggers and Symptoms. J Pers Disord. 2015 Aug;29(4): 486-502.

Murrell AR, Al-Jabari R, Moyer DN, Novamo E, Connally ML. An acceptance and commitment therapy approach to adolescent suicide. J Behav Consult Ther. 2014 Jan;9(3):41-6.

Nock MK, Prinstein MJ. A functional approach to the assessment of self-mutilative behavior. J Consult Clin Psychol. 2004 Oct;72(5):885-90.

O'Connor RC. The integrated motivational-volitional model of suicidal behavior. Crisis. 2011;32(6):295-8.

Oumaya M, Friedman S, Pham A, Abou Abdallah T, Guelfi JD, Rouillon F. [Borderline personality disorder, self-mutilation and suicide: literature review]. Encephale. 2008 Oct;34(5):452-8. French.

Paashaus L, Forkmann T, Glaesmer H, Juckel G, Rath D, Schönfelder A, et al. Do suicide attempters and suicide ideators differ in capability for suicide? Psychiatry Res. 2019 May; 275:304-9.

Paris J. Half in love with easeful death: the meaning of chronic suicidality in borderline personality disorder. Harv Rev Psychiatry. 2004 Jan-Feb;12(1):42-8.

Paris J. Suicidality in Borderline Personality Disorder. Medicina (Kaunas). 2019 May;55(6): E223.

Paris J, Zweig-Frank H. A 27-year follow-up of patients with borderline personality disorder. Compr Psychiatry. 2001 Nov-Dec;42(6): 482-7.

Pfennig A, Kunzel HE, Kern N, Ising M, Majer M, Fuchs B, et al. Hypothalamus-pituitary-adrenal system regulation and suicidal behavior in depression. Biol Psychiatry. 2005 Feb;57(4): 336-42.
Plener PL, Libal G, Keller F, Fegert JM, Muehlenkamp JJ. An international comparison of adolescent non-suicidal self-injury (NSSI) and suicide attempts: Germany and the USA. Psychol Med. 2009 Sep;39(9):1549-58.

Prinstein MJ, Heilbron N, Guerry JD, Franklin JC, Rancourt D, Simon V, et al. Peer influence and nonsuicidal self injury: longitudinal results in community and clinically-referred adolescent samples. J Abnorm Child Psychol. 2010 Jul;38(5):669-82.

Reisch T, Seifritz E, Esposito F, Wiest R, Valach L, Michel K. An fMRI study on mental pain and suicidal behavior. J Affect Disord. 2010 Oct; 126(1-2):321-5

Reitz S, Kluetsch R, Niedtfeld I, Knorz T, Lis S, Paret $\mathrm{C}$, et al. Incision and stress regulation in borderline personality disorder: neurobiological mechanisms of self-injurious behaviour. Br J Psychiatry. 2015 Aug;207(2):165-72.

Ren Y, You J, Zhang X, Huang J, Conner BT, Sun $\mathrm{R}$, et al. Differentiating Suicide Attempters from Suicide Ideators: The Role of Capability for Suicide. Arch Suicide Res. 2019 Jan-Mar; 23(1):64-81.

Robinson J, Hill NT, Thorn P, Battersby R, Teh Z, Reavley NJ, et al. The \#chatsafe project. Developing guidelines to help young people communicate safely about suicide on social media: a Delphi study. PLoS One. 2018 Nov; 13(11):e0206584.

Roy B, Dwivedi Y. Understanding epigenetic architecture of suicide neurobiology: a critical perspective. Neurosci Biobehav Rev. 2017 Jan;72:10-27.

Taiminen TJ, Kallio-Soukainen K, Nokso-Koivisto $\mathrm{H}$, Kaljonen A, Helenius $\mathrm{H}$. Contagion of deliberate self-harm among adolescent inpatients. J Am Acad Child Adolesc Psychiatry. 1998 Feb;37(2):211-7.

Taylor PJ, Jomar K, Dhingra K, Forrester R, Shahmalak U, Dickson JM. A meta-analysis of the prevalence of different functions of non-suicidal self-injury. J Affect Disord. 2018 Feb;227:759-69.

Teismann T, Paashaus L, Siegmann P, Nyhuis P, Wolter M, Willutzki U. Suicide Attempters, Suicide Ideators, and Non-Ideators. Crisis. 2019 Jul;40(4):294-7.

Tullis K. A theory of suicide addiction. Sex Addict Compulsivity. 1998;5(4):311-24.

Wasserman D, Hoven CW, Wasserman C, Wall M, Eisenberg R, Hadlaczky G, et al. Schoolbased suicide prevention programmes: the SEYLE cluster-randomised, controlled trial. Lancet. 2015 Apr;385(9977):1536-44.

Wolfersdorf M. [Suicidality]. Nervenarzt. 2008 Nov;79(11):1319-34. German.

Young JE, Klosko JS, Weishaar ME. Schema therapy: a practitioner's guide. New York: Guilford Press; 2003.

Zanarini MC, Frankenburg FR, Reich DB, Fitzmaurice G, Weinberg I, Gunderson JG. The 10-year course of physically self-destructive acts reported by borderline patients and axis II comparison subjects. Acta Psychiatr Scand. 2008 Mar;117(3):177-84. 